\title{
Mineralogical and chemical attributes of soils from the Brazilian Atlantic
} Forest domain

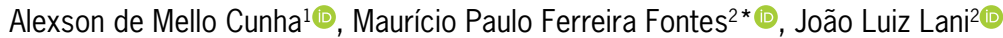

IInstituto Nacional de Colonização e Reforma Agrária/ Superintendência Regional do Espírito Santo, Av. Senador Robert Kennedy, 601 - 29114-300 - Vila Velha, ES - Brasil. ¿Universidade Federal de Viçosa - Depto. de Solos, Av. Peter Henry Rolfs, s/n - 36570-900 - Viçosa, MG - Brasil.

*Corresponding author <mpfontes@ufv.br>

Edited by: Leônidas Carrijo Azevedo Melo

Received March 27, 2017

Accepted August 03, 2017
ABSTRACT: The Atlantic Forest is one of the most important and yet the most devastated Brazilian biome, occupying in the past the majority of the East Atlantic Coast. Therefore, a detailed knowledge of the soils near the coastal line influenced by this biome is essential for land use and management of these environments. The aim of this study was to acquire a deeper knowledge of the chemistry, mineralogy and genesis of soils in two micro-watersheds of the Atlantic Forest Biome. Eight soil profiles were sampled and described along one transect. Both young and more developed soils exhibit low fertility and low $\mathrm{Fe}_{2} \mathrm{O}_{3}$ content. The Oxic Dystrudepts are less dystrophic than the other soils studied and exhibit greater availability of $\mathrm{K}^{+}$, due to the mixing of weathered material and primary minerals. Transitions from shallow to deep soils are more common in rejuvenated landscapes. All soils are kaolinitic (Kt), with low levels of goethite (Gt) and hematite $(\mathrm{Hm})$, and contain secondary 2:1 minerals such as mica (Mi) and, in the youngest soils, hydroxy-interlayered vermiculite (HIV) and interstratified mica-hydroxy-interlayered vermiculite (MiHIV). The widespread presence of gibbsite (Gb) in soils, including the Lithic Udifolist, indicates high rates of both pedogenesis and morphogenesis. The gibbsite showed good crystallinity suggesting that climate, terrain and good drainage favored its formation and stability.

Keywords: Forest biome, coastal plains, coastal uplands, pedogenesis

\section{Introduction}

The Atlantic Forest is the most threatened biome in Brazil (Joly et al., 2014) and the degradation that began in the $16^{\text {th }}$ century accelerated with the expansion of agriculture and the establishment of immigrants along the Brazilian coast (Nettesheim, 2010). The Atlantic Forest today has shrunk to $8 \%$ of its original area, considering fragments over 100 ha, which are important to maintaining its biodiversity, and it continues to suffer severe anthropogenic pressure, risking imminent extinction of the remaining species (Morellato and Haddad, 2000).

Knowledge of the mineral composition and chemical properties of the soil is essential for understanding its genesis and fundamental for assessing land use potential and limitations. This knowledge is also important for the possible restoration of this biome. Robinson et al. (2015) stated that ecologically relevant restoration of the secondary Atlantic forest on abandoned land offers a potential means to recover biodiversity and improve crucial ecosystem functions, including carbon sequestration. However, their results suggest that recovery of secondary forest and below- and aboveground carbon storage is limited by the amount of adjacent forest, certain soil properties such as soil acidity, $\mathrm{P}$ content, clay proportion, and dense shrub establishment, which would down-regulate the succession process.

Research projects in the state of Espírito Santo (ES) involving environmental stratification (Lani et al., 2001) and soil characterization in detail for investigating soil genesis have made progress, mainly in Oxisols and Ultisols on the Coastal Plains (Zangrande and Rezende, 1989) and in a few studies on Histosols in the Quater- nary Plains (Nascimento et al., 2013). Little new knowledge has been added to what is already known about soils in more elevated coastal areas formed on crystalline bedrock. It is on these more elevated parts of the area studied that, in addition to traditional crop and livestock farming, ecotourism and urban expansion are currently growing activities, influenced by the beauty of the landscapes.

In view of the importance of the rational use of natural resources and environmental conservation, the aim of the study was to characterize the soils chemically and mineralogically to deepen the knowledge of genesis and development of the main soil classes in watersheds located in an Atlantic Forest area in Guarapari, ES, Brazil.

\section{Materials and Methods}

\section{Study area and soils collection}

The area is located in Guarapari county, ES, between the parallels $20^{\circ} 33^{\prime}$ and $20^{\circ} 42^{\prime}$ south and meridians $40^{\circ} 31^{\prime}$ and $40^{\circ} 37^{\prime}$ west. The areas studied are in the micro-watersheds of the Oratório and Limão streams in the Guarapari river basin. The Köppen climate classifications are Aw - hot and humid with a rainy season on the coastal lowlands (coastal plains), and Am - hot and humid with a poorly defined dry season on the uplands and slopes above $300 \mathrm{~m}$. Elevations vary from sea level to $775 \mathrm{~m}$ and total average annual rainfall from 1,000 $\mathrm{mm}$ to $1,300 \mathrm{~mm}$ (Embrapa, 1978).

Most of the area consists of garnetiferous gneiss and kinzigites (South Paraiba Complex, and to a lesser degree, Espírito Santo Intrusive Suite) and a narrow 
strip of Tertiary sediments (Barreiras Formation), which sometimes come into contact with the sea (Embrapa, 1978; MME, 1983). The geomorphology of the area comprises the coastal uplands and mountains, and the coastal plains, bordered by the coastal uplands and mountains to the west and by the sea to the east (MME, 1983).

Eight profiles of soils occurring frequently in the micro-watersheds studied were selected, six in the Crystalline Complex and two in the Barreiras Formation sediments (Figure 1). Although the original natural vegetation in the area is Atlantic Forest, the soils studied were under various uses (grassland, banana and coffee plantations). The soil profiles were described morphologically and sampled according to Santos et al. (2005). The soils were classified according to the Brazilian Soil Classification System and their U.S. Soil Taxonomy (Soil Survey Staff, 2014) approximation is shown in Table 1. The soils were described in mini-trenches and in deeply prepared road cuts of vicinal roads (Santos et al., 2005), all of them located along an east west transect, consisting of parts of the Barreiras Group and the Crystalline Complex. Soil samples were collected from the surface and subsurface diagnostic horizons of each profile studied. They were taken to the laboratory, passed through a $2 \mathrm{~mm}$ sieve to make up the fine earth (FE), and were utilized for chemical and mineralogical analyses.

\section{Chemical characterization}

Chemical characterization consisted of determining exchangeable calcium, magnesium and aluminum extracted in a $1 \mathrm{~mol} \mathrm{~L}^{-1} \mathrm{KCl}$ solution and titrated; potassium and sodium were extracted in a Mehlich-1 solution and subjected to flame photometry; available phosphorus was extracted in a Mehlich-1 solution and subjected to colorimetry, and $\mathrm{pH}$ (in water and in a 1 mol L ${ }^{-1} \mathrm{KCl}$ solution). All procedures were compliant with Embrapa (1997). Organic carbon was determined using the Walkley and Black method (Walkely and Black, 1934). Remaining phosphorus (Prem) was analyzed by colorimetry after reacting the samples with a solution of $60 \mathrm{mg} \mathrm{L}^{-1}$ of $\mathrm{P}$.

Sulfuric acid digestion $\mathrm{H}_{2} \mathrm{SO}_{4}(1: 1 \mathrm{v} / \mathrm{v})$ enabled the elemental composition of the clay fraction, in oxide form, to be calculated from the determination of the following elements: $\mathrm{Si}, \mathrm{Al}, \mathrm{Fe}, \mathrm{Ti}, \mathrm{K}$ and $\mathrm{P}$ (Embrapa, 1997).

\section{Mineralogical characterization}

Mineralogical characterization consisted of the determination of iron oxide contents and X-ray Diffraction Analysis (XRD).

In the clay fraction, amorphous iron oxide contents were determined, after extraction with $0.2 \mathrm{~mol} \mathrm{~L}^{-1}$

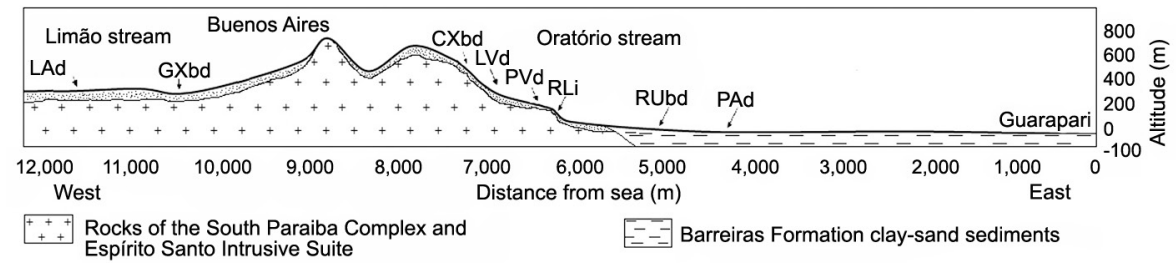

Figure 1 - Elevation profile obtained by GPS and diagram of the marine transect up to Córrego do Limao micro-watershed, showing profile description and locations of the soil sample collection.

Table 1 - Classification of soil profiles in the Brazilian system, its approximation to Soil Taxonomy, location and environment characteristics.

\begin{tabular}{|c|c|c|c|c|c|}
\hline \multirow{2}{*}{$\begin{array}{l}\text { Soil } \\
\text { Profile } \\
\end{array}$} & \multicolumn{2}{|c|}{ Soil Classification System } & \multicolumn{2}{|c|}{ UTM Coordinates $^{1}$} & \multirow{2}{*}{ Environment characteristics } \\
\hline & Brazilian (Embrapa, 2013) & Soil Taxonomy (Soil Survey Staff, 2014) & $\mathrm{N}$ & $\mathrm{E}$ & \\
\hline RLi & Neossolo Litólico Distrófico hístico & Lithic Udifolist & 7720405 & 339978 & $\begin{array}{l}\text { Mountainous relief, lower third of the slope, } \\
\text { originated from gneiss. Altitude: } 91 \mathrm{~m}\end{array}$ \\
\hline PVd & Argissolo Vermelho Distrófico típico & Typic Paleudult & 7720663 & 339905 & $\begin{array}{l}\text { Strong wavy relief, lower third of the slope, } \\
\text { originated from gneiss. Altitude: } 132 \mathrm{~m}\end{array}$ \\
\hline LVd & Latossolo Vermelho Distrófico típico & Typic Hapludox & 7720913 & 339853 & $\begin{array}{l}\text { Strong wavy relief, middle third of the slope, } \\
\text { originated from gneiss. Altitude: } 176 \mathrm{~m}\end{array}$ \\
\hline CXbd & Cambissolo Háplico Tb Distrófico típico & Oxic Dystrudept & 7721574 & 339804 & $\begin{array}{l}\text { Mountainous relief, upper third of the slope, } \\
\text { originated from gneiss. Altitude: } 247 \mathrm{~m}\end{array}$ \\
\hline RYbd & Neossolo Flúvico Tb Distrófico típico & Oxyaquic Udifluvent & 7719395 & 340765 & $\begin{array}{l}\text { Flat relief, larger streambed, originated from } \\
\text { fluvial sediments. Altitude: } 10 \mathrm{~m}\end{array}$ \\
\hline PAd & Argissolo Amarelo Distrófico Latossólico & Typic Hapludult & 7718669 & 340807 & $\begin{array}{l}\text { Mountainous relief, lower third of the slope, } \\
\text { originated of Tertiary sediments. Altitude: } 15 \mathrm{~m}\end{array}$ \\
\hline GXbd & Gleissolo Háplico Tb Distrófico típico & Typic Endoaquent & 7722984 & 339560 & $\begin{array}{l}\text { Flat relief, lowland, originated from alluvial } \\
\text { sediments. Altitude: } 305 \mathrm{~m}\end{array}$ \\
\hline LAd & Latossolo Amarelo Distrófico típico & Xanthic Hapludox & 7726521 & 337860 & $\begin{array}{l}\text { Mountainous relief, lower third of the slope, } \\
\text { originated from gneiss. Altitude: } 359 \mathrm{~m}\end{array}$ \\
\hline
\end{tabular}

IZone 24, Datum Sirgas, 2000. 
acid-ammonium oxalate (McKeague and Day, 1966), and the crystalline iron oxides were determined after extraction with $0.2 \mathrm{~mol} \mathrm{~L}^{-1}$ dithionite citrate solution (Coffin, 1963). The values obtained in the clay fraction were corrected for the fine earth to improve the evaluation of $\mathrm{Fe}_{2} \mathrm{O}_{3}$ content ratios.

For the X-ray diffraction technique (Whittig and Allardice, 1986), the organic matter in the soil samples was oxidized (Anderson, 1963), the clay fraction separated (Jackson, 1979) and the iron oxides removed from this fraction using dithionite citrate treatment (Coffin, 1963). Slides were prepared from natural unspoilt samples (without iron oxide removal) and slides with the iron oxides removed were solvated using $\mathrm{K}$ and $\mathrm{Mg}$, when necessary, and then irradiated at an angle interval $(2 \theta)$ between 4 and $44^{\circ}$ with a goniometer speed of $2^{\circ} 2 \theta \mathrm{min}^{-1}$ using Co$\mathrm{K} \alpha$ radiation. For thorough identification of the clay minerals, a number of samples saturated with $\mathrm{K}$ were heated to $300{ }^{\circ} \mathrm{C}$ and $500{ }^{\circ} \mathrm{C}$, and others saturated with $\mathrm{Mg}$ were treated with glycerol before X-ray irradiation. Clay minerals were identified using the obtained reflections of key lines (Chen, 1977). Quantifying the mineral constituents of the clay fraction was based on sulfuric acid attack data using the allocation method (Resende et al., 1987).

\section{Results}

\section{Chemical characteristics}

Results for chemical characteristics of the main diagnostic horizons are presented in Table 2. In general, the soils were acid, categorized between high active acidity $(4.5 \mathrm{pH}>5.0)$ and weak acidity $(5.1<\mathrm{pH}>$ 6.0). The Oxic Dystrudept (CXbd) and the Typic Endoaquent (GXbd) profiles showed, on average, the highest $\mathrm{pH}$ values, whereas the Typic Hapludox (LVd), Xanthic Hapludox (LAd), Typic Paleudult (PVd) and Typic Hapludult (PAd) profiles showed lower $\mathrm{pH}$ values. In terms of $\Delta \mathrm{pH}$, all soils exhibited a predominance of negative liquid charges.

The soil CXbd, due to its higher contents of $\mathrm{Ca}^{2+}$ and especially $\mathrm{Mg}^{2+}$, showed the highest base saturation value. These Oxic Dystrudept, in which levels of $\mathrm{Ca}^{2+}$ are considered good and $\mathrm{Mg}^{2+}$ and $\mathrm{K}^{+}$very good (CFSMG, 1999), are wisely preferred by local family farmers for use with banana plantations, especially in the Oratório stream location.

In the $\mathrm{A}$ and $\mathrm{B}$ horizons of the profiles studied, available phosphorus levels generally varied from low to very low (CFSMG, 1999), except in the Typic Endoaquent (RYbd) profile, which showed a tendency towards higher phosphorus availability. Soil profiles of GXbd, LAd, PAd, PVd, LVd and RLi (Lithic Udifolist) showed the lowest figures for surface Prem, which is a proxy of the power of the soils to adsorb phosphates.

The majority of the soils presented exchangeable $\mathrm{Al}^{3+}$ levels in the range of low to average (CFSMG, 1999). In general, the lower levels of this ion were found closer to the soil surface.

\section{Sulfuric acid digestion}

Jenny (1941) summarized the concepts of $\mathrm{H}$. Harrassovitz and C.F. Marbut of ki or sa and silica-sesquioxides molecular ratios. Then, the $\mathrm{Ki}=\mathrm{SiO}_{2} / \mathrm{Al}_{2} \mathrm{O}_{3}$ of the clay fraction was calculated from the sulfuric acid digestion data and used to estimate the extent of weathering and development of the soils. Results showed that most Ki values were below 2 (Table 3) for the subsurface diagnostic horizons of the soils. The lowest Ki values, around 1 were found, as expected, in the Oxisol and Ultisol profiles (LAd, PAd and LVd).

The RLi, LVd, CXbd, RYbd, GXbd and LAd profiles exhibited diagnostic horizons with $\mathrm{Fe}_{2} \mathrm{O}_{3}$ levels below $8 \mathrm{dag} \mathrm{kg}^{-1}$ and were classified as hypoferric (Embrapa, 2013). The mesoferric soils (PVd and PAd) exhibited levels slightly above 8 dag $\mathrm{kg}^{-1} \mathrm{Fe}_{2} \mathrm{O}_{3}$.

The highest total levels of potassium were found in the CXbd, RYbd and GXbd profiles, reflecting the contribution of micaceous minerals to these soils, whereas total $\mathrm{P}$ levels were relatively low and similar in all the soils studied.

\section{Mineralogical characteristics}

\section{Iron extracted using dithionite and oxalate}

The highest level of Fe extracted using ammonium oxalate, with a correction for fine earth (FE), and the highest iron-oxalate/iron-dithionite (Feo/Fed) ratio (Table 4) was found at the gleyic $\mathrm{C}$ horizon $(\mathrm{Cg})$ of the Typic Endoaquent (GXbd) profile, indicating that this soil has the lowest degree of Fe oxide crystallinity.

At the subsurface horizons of one Typic Hapludox (LVd) and one Typic Paleudult (PVd) profiles, soils with good drainage characteristics, levels of crystalline $\mathrm{Fe}$ oxides were relatively high, a result to be expected in view of the relatively more clayey texture of these profiles. The surface horizons of Paleudult (PVd), Hapludult (PAd), Dystrudept (CXbd) and Xanthic Hapludox (LAd) profiles showed a tendency for the Feo/Fed ratio to increase, evidencing the effect of the organic matter (Table 4). On the other hand, this was not true of the surface horizons of the Typic Hapludox (LVd) and Endoaquent (GXbd) profiles, reflecting the lower differentiation of the total $\mathrm{Fe}_{2} \mathrm{O}_{3}$ level in the horizons of these two profiles.

Soils with good drainage such as the Typic Paleudult, Oxisols and a Dystrudept (PVd, LVd, CXbd, PAd and LAd) exhibited similar Feo/Fed ratios, with the lowest figure for the LAd and PAd profiles, indicating higher levels of wellcrystallized iron oxide, which suggests higher soil weathering and development in these two profiles.

\section{Clay fraction mineralogy}

The clay fraction mineralogical composition (Table 4) based on the X-ray diffraction patterns (Figures 2 and 3) showed that the soils presented consistently kaolinite, goethite and gibbsite as constituent minerals. However, from the patterns, two groups of soils with relatively similar mineral contents were observed. The first group 
Table 2 - Results of soil chemical analyses.

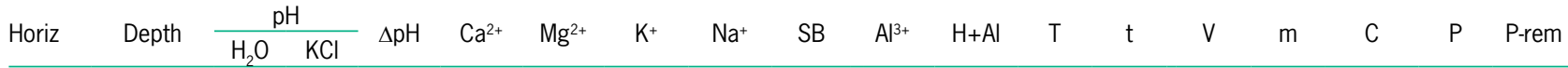
$\mathrm{cm} \quad-\mathrm{cmol} \mathrm{dm}^{-3} \longrightarrow \mathrm{mg} \mathrm{dm}^{-3}-$

\begin{tabular}{|c|c|c|c|c|c|c|c|c|c|c|c|c|c|c|c|c|c|c|}
\hline \multicolumn{5}{|c|}{$\mathrm{cm}$} & \multicolumn{14}{|c|}{$-\mathrm{cmol}_{\mathrm{c}} \mathrm{dm}^{-3}-$} \\
\hline \multicolumn{19}{|c|}{ RLi - Lithic Udifolist } \\
\hline 0 & $0-10$ & 5.2 & 4.2 & -1.0 & 0.2 & 0.3 & 0.06 & 0.02 & 0.5 & 1.0 & 15.5 & 17.64 & 1.5 & 12 & 6 & 12.99 & 5 & 6.6 \\
\hline \multicolumn{19}{|c|}{ PVd - Typic Paleudult } \\
\hline Ap1 & $0-2$ & 6.0 & 4.9 & -1.1 & 1.3 & 0.8 & 0.16 & 0.01 & 2.2 & 0.0 & 1.3 & 1.8 & 2.2 & 29 & 0 & 3.38 & 9 & 47.2 \\
\hline Ap2 & $2-12$ & 5.1 & 4.0 & -1.1 & 0.6 & 0.6 & 0.20 & 0.01 & 1.4 & 0.7 & 3.4 & 4.8 & 2.1 & 28 & 16 & 2.57 & 5 & 27.1 \\
\hline E & $12-24$ & 4.8 & 3.9 & -0.9 & 0.3 & 0.4 & 0.14 & 0.01 & 0.8 & 1.1 & 3.5 & 4.4 & 1.9 & 20 & 25 & 2.17 & 4 & 24.5 \\
\hline BA & $24-42$ & 4.8 & 3.9 & -0.9 & 0.4 & 0.4 & 0.13 & 0.01 & 0.9 & 1.2 & 3.6 & 4.5 & 2.1 & 20 & 26 & 1.90 & 4 & 19.6 \\
\hline Bt1 & $42-80$ & 4.9 & 4.0 & -0.9 & 0.6 & 0.5 & 0.06 & 0.02 & 1.1 & 1.3 & 4.1 & 5.3 & 2.4 & 22 & 26 & 1.76 & 4 & 8.3 \\
\hline Bt2 & $80-122$ & 4.8 & 4.0 & -0.8 & 0.4 & 0.5 & 0.03 & 0.02 & 0.9 & 1.5 & 4.3 & 5.3 & 2.4 & 17 & 30 & 1.49 & 3 & 4.5 \\
\hline $\mathrm{BC}$ & $122-150$ & 4.9 & 4.0 & -0.9 & 0.4 & 0.5 & 0.03 & 0.02 & 1.0 & 1.4 & 3.9 & 4.9 & 2.4 & 19 & 29 & 1.22 & 3 & 4.0 \\
\hline$C$ & $150-185+$ & 5.0 & 4.1 & -0.9 & 0.4 & 0.6 & 0.04 & 0.01 & 1.0 & 1.0 & 3.4 & 4.4 & 2.0 & 21 & 23 & 0.29 & 4 & 6.1 \\
\hline $\mathrm{Cr}$ & $450-480+$ & 5.3 & 4.0 & -1.3 & 0.2 & 0.7 & 0.03 & 0.02 & 0.9 & 1.2 & 2.8 & 3.7 & 2.1 & 24 & 32 & 0.29 & 4 & 23.5 \\
\hline \multicolumn{19}{|c|}{ LVd - Typic Hapludox } \\
\hline Ap & $0-12$ & 5.7 & 4.5 & -1.2 & 1.7 & 1.1 & 0.22 & 0.02 & 3.0 & 0.2 & 5.1 & 8.1 & 3.2 & 37 & 2 & 3.79 & 5 & 22.4 \\
\hline BA & $12-27$ & 4.9 & 4.1 & -0.8 & 0.2 & 0.4 & 0.07 & 0.01 & 0.6 & 1.0 & 4.4 & 5.1 & 1.6 & 12 & 20 & 1.76 & 4 & 10.9 \\
\hline Bw1 & $27-62$ & 4.9 & 4.1 & -0.8 & 0.2 & 0.3 & 0.04 & 0.01 & 0.5 & 0.4 & 5.2 & 5.7 & 0.9 & 9 & 7 & 1.49 & 4 & 12.6 \\
\hline Bw2 & $62-125$ & 5.1 & 4.2 & -0.9 & 0.3 & 0.4 & 0.04 & 0.01 & 0.7 & 0.5 & 3.1 & 3.8 & 1.3 & 18 & 14 & 1.36 & 4 & 8.6 \\
\hline Bw3 & $125-163$ & 4.8 & 4.1 & -0.7 & 0.1 & 0.3 & 0.02 & 0.01 & 0.4 & 0.9 & 3.2 & 3.6 & 1.3 & 11 & 26 & 1.36 & 3 & 6.6 \\
\hline Bw4 & $163-220+$ & 4.9 & 4.1 & -0.8 & 0.2 & 0.1 & 0.08 & 0.05 & 0.4 & 0.6 & 2.8 & 3.2 & 1.0 & 16 & 21 & 0.94 & 5 & 7.9 \\
\hline \multicolumn{19}{|c|}{ CXbd - Oxic Dystrudept } \\
\hline Ap & $0-12$ & 6.5 & 5.3 & -1.2 & 4.7 & 2.3 & 0.46 & 0.02 & 7.5 & 0.0 & 2.7 & 10.2 & 7.5 & 73 & 0 & 3.66 & 7 & 31.9 \\
\hline$A B$ & $12-28$ & 5.8 & 4.6 & -1.2 & 2.3 & 1.4 & 0.28 & 0.01 & 3.9 & 0.1 & 3.6 & 7.5 & 4.0 & 52 & 1 & 2.16 & 5 & 23.0 \\
\hline BA & $28-40$ & 5.3 & 4.3 & -1.0 & 1.4 & 0.7 & 0.23 & 0.01 & 2.3 & 0.2 & 3.5 & 5.9 & 2.6 & 40 & 4 & 1.62 & 4 & 23.3 \\
\hline Bil & $40-60$ & 5.5 & 4.5 & -1.0 & 1.6 & 0.6 & 0.18 & 0.01 & 2.3 & 0.1 & 3.1 & 5.5 & 2.5 & 43 & 3 & 1.62 & 4 & 14.9 \\
\hline Bi2 & $60-80$ & 5.7 & 4.7 & -1.0 & 1.4 & 0.5 & 0.15 & 0.01 & 2.1 & 0.1 & 2.3 & 4.4 & 2.2 & 47 & 2 & 1.36 & 4 & 11.0 \\
\hline Bi3 & $80-110+$ & 5.7 & 4.8 & -0.9 & 1.3 & 0.5 & 0.09 & 0.02 & 1.9 & 0.1 & 2.3 & 4.2 & 2.0 & 45 & 2 & 1.36 & 4 & 9.1 \\
\hline \multicolumn{19}{|c|}{ RYbd - Oxyaquic Udifluvent } \\
\hline Ap1 & $0-10$ & 5.5 & 4.5 & -1.0 & 1.5 & 0.9 & 0.22 & 0.03 & 2.6 & 0.1 & 2.8 & 5.5 & 2.75 & 47 & 3 & 2.03 & 14 & 35.2 \\
\hline Ap2 & $10-18$ & 5.7 & 4.6 & -1.1 & 3.1 & 1.5 & 0.08 & 0.04 & 4.7 & 0.1 & 5.6 & 10.3 & 4.77 & 45 & 0 & 3.66 & 10 & 21.3 \\
\hline $\mathrm{C} 1$ & $18-29$ & 5.0 & 4.1 & -0.9 & 1.3 & 0.7 & 0.05 & 0.03 & 2.1 & 0.7 & 8.9 & 11.0 & 2.83 & 19 & 7 & 3.79 & 9 & 6.7 \\
\hline $\mathrm{C} 2$ & $29-40$ & 5.1 & 4.1 & -1.0 & 0.6 & 0.4 & 0.02 & 0.02 & 1.0 & 0.4 & 4.6 & 5.6 & 1.39 & 18 & 7 & 1.76 & 9 & 18.5 \\
\hline C3 & $40-46$ & 5.4 & 4.2 & -1.2 & 0.6 & 0.4 & 0.02 & 0.02 & 1.0 & 0.3 & 1.9 & 2.9 & 1.34 & 34 & 12 & 0.95 & 13 & 32.4 \\
\hline $\mathrm{Cg}$ & $46-69+$ & 5.5 & 4.1 & -1.4 & 1.5 & 0.7 & 0.03 & 0.03 & 2.2 & 0.1 & 6.0 & 8.2 & 2.31 & 27 & 1 & 2.57 & 11 & 13.9 \\
\hline \multicolumn{19}{|c|}{ PAd - Typic Hapludult } \\
\hline Ap1 & $0-13$ & 5.6 & 4.6 & -1.0 & 1.6 & 1.1 & 0.13 & 0.01 & 2.8 & 0.1 & 3.4 & 6.3 & 2.94 & 45 & 2 & 3.52 & 4 & 24.1 \\
\hline Ap2 & $13-25$ & 5.5 & 4.3 & -1.2 & 0.8 & 0.7 & 0.06 & 0.01 & 1.5 & 0.3 & 4.3 & 5.9 & 1.87 & 26 & 6 & 3.11 & 4 & 30.1 \\
\hline$A B$ & $25-38$ & 5.4 & 4.3 & -1.1 & 0.8 & 1.0 & 0.05 & 0.01 & 1.8 & 0.4 & 4.3 & 4.7 & 2.16 & 37 & 8 & 2.71 & 3 & 22.8 \\
\hline Bt1 & $38-60$ & 5.3 & 4.4 & -0.9 & 0.3 & 0.4 & 0.02 & 0.01 & 0.7 & 0.3 & 2.9 & 3.7 & 1.03 & 19 & 10 & 1.90 & 2 & 12.1 \\
\hline Bt2 & $60-87$ & 5.2 & 4.4 & -0.8 & 0.2 & 0.4 & 0.02 & 0.01 & 0.6 & 0.4 & 2.7 & 3.3 & 1.03 & 18 & 14 & 1.36 & 2 & 7.6 \\
\hline Bt3 & $87-140$ & 5.1 & 4.4 & -0.7 & 0.3 & 0.3 & 0.02 & 0.01 & 0.6 & 0.3 & 2.7 & 3.3 & 0.93 & 18 & 10 & 1.07 & 4 & 6.0 \\
\hline Bt4 & $140-210+$ & 5.2 & 4.7 & -0.5 & 0.2 & 0.2 & 0.01 & 0.01 & 0.4 & 0.1 & 1.9 & 2.3 & 0.47 & 16 & 4 & 1.07 & 4 & 1.4 \\
\hline \multicolumn{19}{|c|}{ GXbd - Typic Endoaquent } \\
\hline Ap1 & $0-17$ & 5.7 & 4.2 & -1.5 & 0.8 & 0.4 & 0.03 & 0.06 & 1.2 & 1.1 & 11.5 & 12.8 & 2.39 & 10 & 9 & 5.19 & 4 & 5.3 \\
\hline Cg1 & $17-37$ & 5.5 & 4.3 & -1.2 & 0.3 & 0.3 & 0.07 & 0.07 & 0.7 & 0.9 & 6.5 & 7.3 & 1.64 & 10 & 12 & 1.45 & 5 & 7.5 \\
\hline $\mathrm{Cg} 2$ & $37-65+$ & 5.7 & 4.3 & -1.4 & 0.3 & 0.2 & 0.03 & 0.03 & 0.5 & 0.6 & 4.2 & 4.8 & 1.16 & 11 & 13 & 1.08 & 4 & 7.0 \\
\hline \multicolumn{19}{|c|}{ LAd - Xanthic Hapludox } \\
\hline Ap & $0-25$ & 5.7 & 4.5 & -1.2 & 0.8 & 0.6 & 0.28 & 0.02 & 1.6 & 0.2 & 3.1 & 4.7 & 1.80 & 34 & 4 & 1.36 & 3 & 24.5 \\
\hline BA & $25-52$ & 5.0 & 4.3 & -0.7 & 0.3 & 0.2 & 0.07 & 0.01 & 0.6 & 0.3 & 2.6 & 3.2 & 0.88 & 17 & 9 & 1.36 & 3 & 13.6 \\
\hline Bw1 & $52-89$ & 5.0 & 4.4 & -0.6 & 0.3 & 0.1 & 0.05 & 0.01 & 0.5 & 0.3 & 2.1 & 2.6 & 0.76 & 18 & 11 & 0.81 & 3 & 10.1 \\
\hline Bw2 & $89-122$ & 5.0 & 4.3 & -0.7 & 0.2 & 0.2 & 0.03 & 0.01 & 0.4 & 0.5 & 3.1 & 3.6 & 0.94 & 12 & 14 & 0.68 & 21 & 14.6 \\
\hline Bw3 & $122-173$ & 4.7 & 4.2 & -0.5 & 0.1 & 0.1 & 0.01 & 0.01 & 0.2 & 0.5 & 2.3 & 2.5 & 0.77 & 9 & 22 & 0.41 & 3 & 6.4 \\
\hline Bw4 & $173-220+$ & 4.5 & 4.1 & -0.4 & 0.1 & 0.1 & 0.02 & 0.01 & 0.2 & 0.7 & 2.8 & 3.0 & 0.98 & 8 & 25 & 0.14 & 2 & 8.1 \\
\hline
\end{tabular}


Table 3 - Results for $\mathrm{SiO}_{2}, \mathrm{Al}_{2} \mathrm{O}_{3}, \mathrm{Fe}_{2} \mathrm{O}_{3}, \mathrm{TiO}_{2}, \mathrm{~K}_{2} \mathrm{O}, \mathrm{MnO}$ and $\mathrm{P}_{2} \mathrm{O}_{5}$ obtained by sulfuric acid digestion on fine earth (FE) and $\mathrm{Ki}$.

\begin{tabular}{|c|c|c|c|c|c|c|c|c|c|}
\hline Horiz. & Depth & $\mathrm{SiO}_{2}$ & $\mathrm{Al}_{2} \mathrm{O}_{3}$ & $\mathrm{Fe}_{2} \mathrm{O}_{3}$ & $\mathrm{TiO}_{2}$ & $\mathrm{~K}_{2} \mathrm{O}$ & $\mathrm{P}_{2} \mathrm{O}_{5}$ & $\mathrm{MnO}$ & $\mathrm{Ki}$ \\
\hline & $\mathrm{cm}$ & \multicolumn{8}{|c|}{ dag kg $\mathrm{kg}^{-1}$} \\
\hline \multicolumn{10}{|c|}{ RLi - Lithic Udifolist } \\
\hline$\underline{0}$ & $0-10$ & 20.0 & 18.0 & 4.28 & 0.66 & 0.08 & 0.14 & 45 & 1.88 \\
\hline \multicolumn{10}{|c|}{ PVd - Typic Paleudult } \\
\hline Ap1 & $0-2$ & 10.6 & 6.9 & 2.14 & 0.31 & 0.13 & 0.06 & 48 & 2.62 \\
\hline Ap2 & $2-12$ & 25.4 & 13.7 & 6.77 & 0.48 & 0.18 & 0.07 & 37 & 3.14 \\
\hline $\mathrm{E}$ & $12-24$ & 18.6 & 17.0 & 3.94 & 0.63 & 0.17 & 0.05 & 24 & 1.86 \\
\hline BA & $24-42$ & 20.0 & 18.0 & 4.83 & 0.83 & 0.17 & 0.05 & 21 & 1.88 \\
\hline Bt1 & $42-80$ & 18.1 & 31.7 & 8.17 & 1.35 & 0.18 & 0.08 & 33 & 0.97 \\
\hline $\mathrm{Bt} 2$ & $80-122$ & 26.7 & 32.1 & 8.45 & 1.31 & 0.20 & 0.08 & 37 & 1.41 \\
\hline $\mathrm{BC}$ & $122-150$ & 18.1 & 20.0 & 7.79 & 1.25 & 0.18 & 0.08 & 83 & 1.53 \\
\hline $\mathrm{C}$ & $150-185+$ & 19.6 & 23.1 & 5.70 & 1.05 & 0.18 & 0.09 & 160 & 1.44 \\
\hline $\mathrm{Cr}$ & $450-480+$ & 24.1 & 26.9 & 9.38 & 1.38 & 0.39 & 0.08 & 50 & 1.52 \\
\hline \multicolumn{10}{|c|}{ LVd - Typic Hapludox } \\
\hline Ap & $0-12$ & 22.3 & 20.9 & 7.51 & 1.23 & 0.11 & 0.11 & 27 & 1.81 \\
\hline BA & $12-27$ & 22.4 & 28.0 & 8.16 & 1.33 & 0.11 & 0.08 & 20 & 1.36 \\
\hline Bw1 & $27-62$ & 24.6 & 27.7 & 8.12 & 1.28 & 0.09 & 0.09 & 21 & 1.51 \\
\hline Bw2 & $62-125$ & 22.4 & 32.0 & 7.95 & 1.22 & 0.09 & 0.09 & 20 & 1.19 \\
\hline Bw3 & $125-163$ & 24.8 & 27.8 & 7.57 & 1.20 & 0.07 & 0.08 & 18 & 1.52 \\
\hline Bw4 & $163-220+$ & 25.1 & 29.1 & 7.58 & 1.20 & 0.09 & 0.08 & 10 & 1.47 \\
\hline \multicolumn{10}{|c|}{ CXbd - Oxic Dystrudept } \\
\hline Ap & $0-12$ & 18.7 & 13.8 & 4.25 & 0.53 & 0.34 & 0.12 & 384 & 2.29 \\
\hline$A B$ & $12-28$ & 17.7 & 16.5 & 4.27 & 0.64 & 0.32 & 0.11 & 455 & 1.82 \\
\hline BA & $28-40$ & 17.3 & 15.7 & 3.80 & 0.56 & 0.27 & 0.09 & 379 & 1.87 \\
\hline Bil & $40-60$ & 20.9 & 17.6 & 4.55 & 0.66 & 0.29 & 0.10 & 365 & 2.01 \\
\hline $\mathrm{Bi} 2$ & $60-80$ & 21.1 & 19.0 & 4.45 & 0.69 & 0.28 & 0.09 & 308 & 1.89 \\
\hline $\mathrm{Bi} 3$ & $80-110+$ & 25.6 & 19.4 & 4.86 & 0.69 & 0.29 & 0.08 & 134 & 2.24 \\
\hline \multicolumn{10}{|c|}{ RYbd - Oxyaquic Udifluvent } \\
\hline Ap1 & $0-10$ & 14.8 & 12.2 & 4.30 & 0.67 & 0.36 & 0.08 & 76 & 2.07 \\
\hline Ap2 & $10-18$ & 24.5 & 21.2 & 6.76 & 1.16 & 0.48 & 0.14 & 148 & 1.96 \\
\hline $\mathrm{C} 1$ & $18-29$ & 26.4 & 26.6 & 7.81 & 1.43 & 0.41 & 0.16 & 109 & 1.68 \\
\hline $\mathrm{C} 2$ & $29-40$ & 19.9 & 18.8 & 5.22 & 1.12 & 0.43 & 0.10 & 66 & 1.79 \\
\hline C3 & $40-46$ & 13.1 & 11.2 & 3.78 & 0.51 & 0.38 & 0.06 & 74 & 1.99 \\
\hline $\mathrm{Cg}$ & $46-49+$ & 16.2 & 20.2 & 6.60 & 1.11 & 0.52 & 0.15 & 121 & 1.36 \\
\hline \multicolumn{10}{|c|}{ PAd - Typic Hapludult } \\
\hline Ap1 & $0-13$ & 19.6 & 22.5 & 6.15 & 1.12 & 0.08 & 0.08 & 26 & 1.48 \\
\hline Ap2 & $13-25$ & 21.5 & 24.2 & 6.90 & 1.35 & 0.08 & 0.08 & 26 & 1.51 \\
\hline$A B$ & $25-38$ & 21.9 & 24.2 & 6.40 & 1.37 & 0.08 & 0.08 & 20 & 1.54 \\
\hline Bt1 & $38-60$ & 18.4 & 24.2 & 8.25 & 1.67 & 0.08 & 0.07 & 17 & 1.29 \\
\hline Bt2 & $60-87$ & 23.41 & 31.4 & 9.48 & 1.57 & 0.08 & 0.11 & 18 & 1.27 \\
\hline Bt3 & $87-140$ & 24.9 & 28.5 & 8.69 & 1.51 & 0.08 & 0.07 & 13 & 1.48 \\
\hline Bt4 & $140-210+$ & 18.0 & 21.8 & 9.64 & 1.64 & 0.09 & 0.08 & 18 & 1.40 \\
\hline \multicolumn{10}{|c|}{ GXbd - Typic Endoaquent } \\
\hline Ap1 & $0-17$ & 24.4 & 25.6 & 6.95 & 1.54 & 0.34 & 0.15 & 28 & 1.62 \\
\hline Ap2 & $17-37$ & 25.1 & 27.6 & 7.96 & 1.53 & 0.43 & 0.11 & 34 & 1.54 \\
\hline$A B$ & $37-65+$ & 16.0 & 24.0 & 7.36 & 1.43 & 0.65 & 0.09 & 28 & 1.13 \\
\hline \multicolumn{10}{|c|}{ LAd - Xanthic Hapludox } \\
\hline Ap & $0-25$ & 17.3 & 18.1 & 6.03 & 1.01 & 0.07 & 0.10 & 83 & 1.62 \\
\hline BA & $25-52$ & 19.3 & 27.0 & 7.46 & 1.35 & 0.07 & 0.11 & 66 & 1.21 \\
\hline Bw1 & $52-89$ & 21.9 & 27.0 & 8.15 & 1.37 & 0.07 & 0.11 & 21 & 1.37 \\
\hline Bw2 & $89-122$ & 16.0 & 24.8 & 7.27 & 1.07 & 0.07 & 0.20 & 21 & 1.10 \\
\hline Bw3 & $122-173$ & 15.9 & 26.1 & 6.84 & 1.05 & 0.07 & 0.09 & 13 & 1.03 \\
\hline Bw4 & $173-220+$ & 27.0 & 28.6 & 7.30 & 1.10 & 0.07 & 0.07 & 26 & 1.60 \\
\hline Bw4 & $173-220+$ & 27.0 & 28.6 & 7.30 & 1.10 & 0.07 & 0.07 & 26 & 1.60 \\
\hline
\end{tabular}




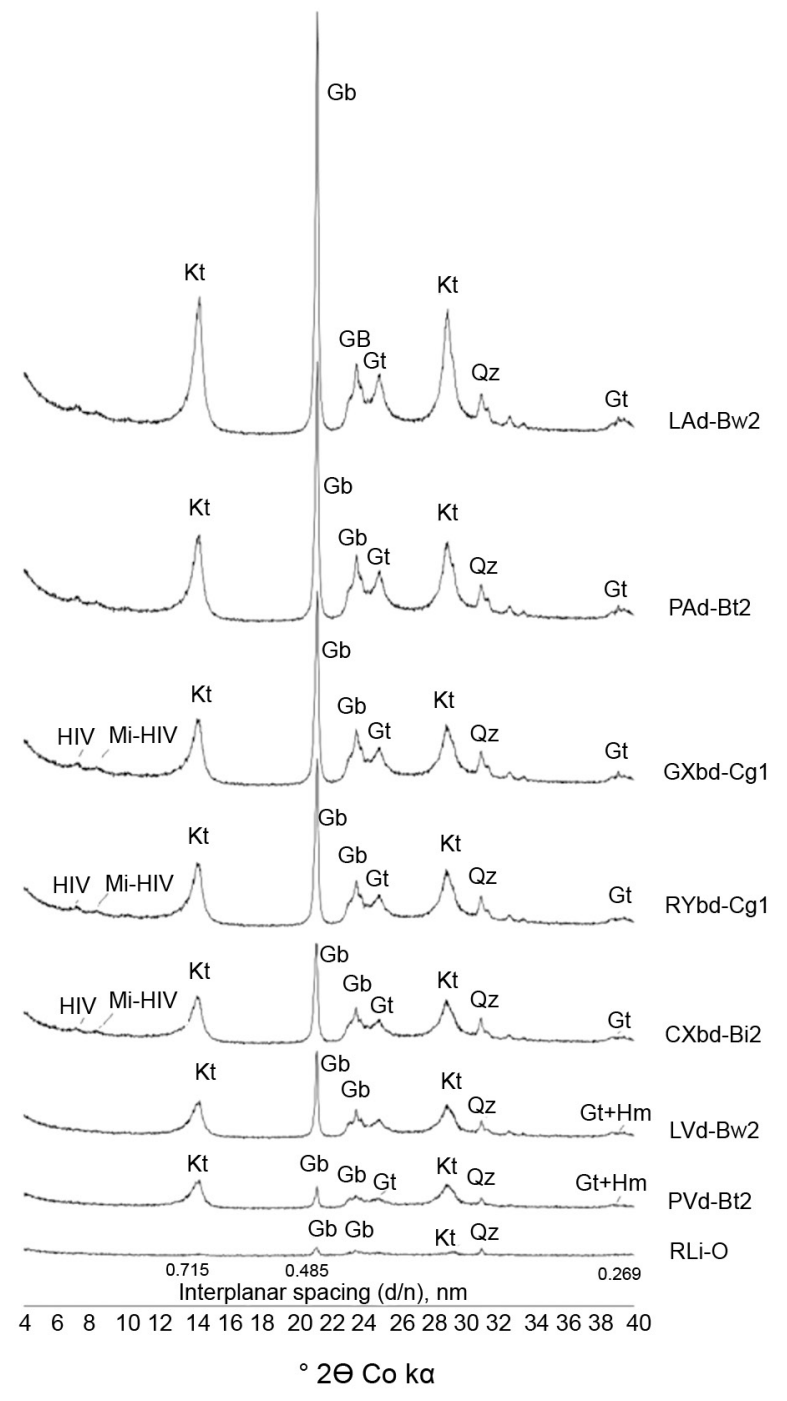

Figure 2 - X-ray diffraction patterns of clay fraction (deferrated, $\mathrm{K}$ solvated and mounted on glass slides) of the profiles RLi = Lithic Udifolist (0), PVd = Typic Paleudult (Bt2), LVd = Typic Hapludox (Bw2), CXbd = Oxic Dystrudept (Bi2), RYbd = Oxyaquic Udifluvent (C1), PAd = Typic Hapludult (Bt2) GXbd = Typic Endoaquent (Cg1) and LAd = Xanthic Hapludox (Bw2). Kt = kaolinite; Gb = gibbsite; Gt = goethite; $\mathrm{Hm}$ = Hematite; Mi-HIV = interstratified mica-hydroxy-interlayered vermiculite; HIV = hydroxy-interlayered vermiculite; $\mathrm{Qz}=$ quartz.

included the more weathered soils, represented by the Ultisol (PVd, PAd) and Oxisol1 (LVd and LAd) profiles. The main minerals in these soils were kaolinite (Kt), gibbsite (Gb) and goethite (Gt). Peaks of hematite ( $\mathrm{Hm})$ were found only in the red soil profiles (PVd and LVd). In this group, small peaks of mica (not shown) appeared in the PVd profile. In addition to the presence of mica (Mi), small peaks of gibbsite were found in the C-horizon of this soil.

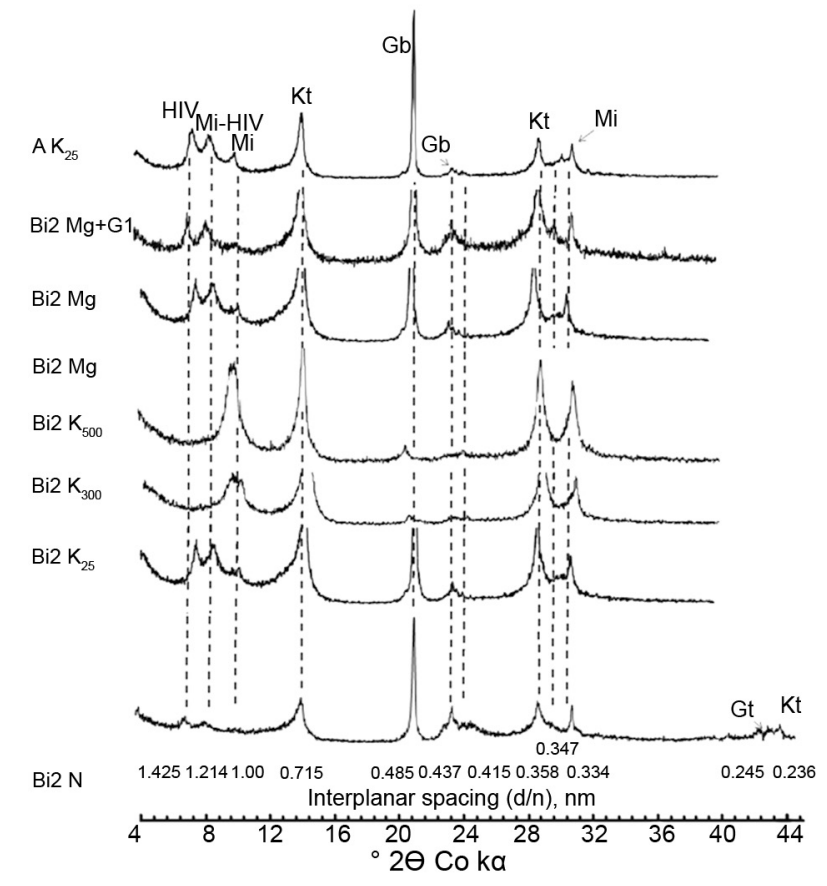

Figure 3 - X-ray patterns of the clay fraction (oriented sample) of the Cambissolo Háplico (CXbd), natural clay (N) saturated with $\mathrm{K}$ at 25,300 and $500{ }^{\circ} \mathrm{C}(\mathrm{K} \mathrm{25}, \mathrm{K} 300$ and $\mathrm{K} \mathrm{500)}$, saturated with $\mathrm{Mg}(\mathrm{Mg})$ and with $\mathrm{Mg}+$ glycerol $(\mathrm{Mg}+\mathrm{Gl})$. Kt = kaolinite; $\mathrm{Gb}=$ gibbsite; $\mathrm{Gt}=$ goethite; $\mathrm{Mi}=$ mica; Mi-HIV = interstratified mica-hydroxy-interlayered vermiculite; HIV = hydroxy-interlayered vermiculite.

The second group of relatively similar soils included the Lithic Udifolist, Udifluvent, Dystrudept and Endoaquent (RLi, RYbd, CXbd and GXbd) profiles, containing 2:1 minerals such as mica, interstratified mica-hydroxyinterlayered vermiculite (Mi-HIV) and hydroxy-interlayered vermiculite (Table 5). However, the small peaks in the XRD patterns seem to indicate low levels of these minerals, with kaolinite persisting as predominant, followed by gibbsite and Fe oxides. X-ray patterns of the Dystrudept (CXbd) show these features in Figure 3.

Semi-quantification of minerals by allocation (Table 6) showed the predominance of kaolinite in all the soil samples, especially in one Hapludult (PAd) and one Paleudult (PVd) profile, the highest ones in kaolinite content. Goethite and gibbsite were present in almost all soils and hematite in a few of them, the reddest ones. The mineralogy as described by the x-ray diffraction agreed with the allocation method.

\section{Discussion}

\section{Chemical characteristics}

The higher $\mathrm{pH}$ of the Dystrudept (CXbd) (Table 2) seems to indicate the release of reasonable amounts of bases from the weathering of the original material closer 
Table 4 - Contents of $\mathrm{Fe}_{2} \mathrm{O}_{3}$ extracted by dithionite citrate (Fed) obtained by four successive extractions and extracted by ammonium oxalate (Feo), Feo/Fed, and Fed/Fes ratios.

\begin{tabular}{|c|c|c|c|c|c|c|c|c|c|c|}
\hline \multirow{3}{*}{ Soil } & \multirow{3}{*}{ Horizon } & \multicolumn{7}{|c|}{ Extraction } & \multicolumn{2}{|c|}{ Ratio } \\
\hline & & \multicolumn{5}{|c|}{$\mathrm{Fed}^{1}$} & \multirow{2}{*}{$\begin{array}{c}\mathrm{Feo} \\
\text { (clay) }^{2}\end{array}$} & \multirow{2}{*}{$\mathrm{FeO}^{3}$} & \multirow{2}{*}{$\mathrm{Feo} / \mathrm{Fed}$} & \multirow{2}{*}{ Fed/ $\mathrm{Fes}^{4}$} \\
\hline & & $1^{\mathrm{st}}$ & $2^{\text {nd }}$ & $3^{\text {rd }}$ & $4^{\text {th }}$ & $\Sigma$ & & & & \\
\hline & & & & & dag $\mathrm{kg}^{-1}$ & & & & & \\
\hline RLi & 0 & 0.58 & 0.13 & 0.11 & 0.07 & 0.89 & 1.19 & 0.15 & 0.17 & 0.21 \\
\hline \multirow[t]{2}{*}{ PVd } & Ap2 & 1.32 & 0.09 & 0.02 & 0.01 & 1.44 & 0.87 & 0.20 & 0.14 & 0.21 \\
\hline & Bt2 & 4.16 & 0.25 & 0.03 & 0.01 & 4.45 & 0.69 & 0.39 & 0.09 & 0.53 \\
\hline \multirow[t]{2}{*}{ LVd } & Ap & 2.54 & 0.58 & 0.26 & 0.09 & 3.47 & 0.63 & 0.24 & 0.07 & 0.46 \\
\hline & Bw2 & 3.74 & 0.78 & 0.26 & 0.13 & 4.91 & 0.76 & 0.37 & 0.08 & 0.62 \\
\hline \multirow[t]{2}{*}{ cXbd } & Ap & 0.88 & 0.09 & 0.10 & 0.02 & 1.09 & 1.04 & 0.18 & 0.17 & 0.26 \\
\hline & Bi2 & 1.48 & 0.14 & 0.04 & 0.02 & 1.68 & 0.74 & 0.16 & 0.10 & 0.38 \\
\hline \multirow[t]{2}{*}{ RYbd } & Ap1 & 0.77 & 0.21 & 0.06 & 0.03 & 1.07 & 0.73 & 0.07 & 0.07 & 0.25 \\
\hline & $\mathrm{C} 1$ & 2.36 & 0.58 & 0.16 & 0.12 & 3.22 & 0.73 & 0.23 & 0.07 & 0.41 \\
\hline \multirow[t]{2}{*}{ PAd } & Ap1 & 1.49 & 0.59 & 0.20 & 0.26 & 2.54 & 0.51 & 0.15 & 0.06 & 0.41 \\
\hline & Bt2 & 3.41 & 1.36 & 1.04 & 0.71 & 6.52 & 0.53 & 0.28 & 0.04 & 0.69 \\
\hline \multirow[t]{2}{*}{ GXbd } & $A p$ & 1.84 & 0.70 & 0.41 & 0.22 & 3.17 & 0.97 & 0.35 & 0.11 & 0.46 \\
\hline & Cg1 & 2.15 & 0.68 & 0.37 & 0.19 & 3.39 & 1.74 & 0.61 & 0.18 & 0.43 \\
\hline \multirow[t]{2}{*}{ LAd } & Ap & 1.04 & 0.65 & 0.38 & 0.09 & 2.16 & 0.72 & 0.16 & 0.07 & 0.36 \\
\hline & Bw2 & 3.21 & 1.15 & 0.35 & 0.12 & 4.83 & 0.57 & 0.20 & 0.04 & 0.66 \\
\hline
\end{tabular}

${ }^{1}$ Level of $\mathrm{Fe}_{2} \mathrm{O}_{3}$ extracted from the clay fraction and corrected for fine earth; ${ }^{2} \mathrm{Level}$ of $\mathrm{Fe}_{2} \mathrm{O}_{3}$ extracted from the clay fraction; ${ }^{3}$ Feo corrected for fine earth; ${ }^{4} \mathrm{Content}$ of $\mathrm{Fe}_{2} \mathrm{O}_{3}$ from the sulfuric acid digestion.

Table 5 - Mineralogical composition by X-ray diffraction of the clay fraction.

\begin{tabular}{lll}
\hline Soil & Horizon & \multicolumn{1}{c}{ Mineral composition } \\
\hline RLi & 0 & Kt, Gt, Gb, Mi, HIV \\
PVd & Bt2 & Kt, Gt, Gb, Hm, Mi \\
LVd & Bw2 & Kt, Gt, Gb, Hm \\
CXbd & Bi2 & Kt, Gt, Gb, Mi, HIV \\
RYbd & C1 & Kt, Gt, Gb, Mi, Mi-HIV, HIV \\
PAd & Bt2 & Kt, Gt, Gb \\
GXbd & Cg1 & Kt, Gt, Gb, Mi, Mi-HIV, HIV \\
LAd & Bw2 & Kt, Gt, Gb \\
\hline
\end{tabular}

$\mathrm{Kt}=$ kaolinite; $\mathrm{Mi}=$ mica; $\mathrm{Gt}=$ goethite; $\mathrm{Hm}=$ hematite; $\mathrm{Gb}=$ gibbsite; Mi-HIV = interstratified mica-hydroxy-interlayered vermiculite; HIV = hydroxyinterlayered vermiculite.

to the surface. Although this soil has latosolic properties, given its typical small granular structure, the presence of rock fragments in the soil mass or at the surface is common and justifies its definition as an Inceptisol.

Albuquerque Filho et al. (2008) working in the Médio Rio Doce region of the state of Minas Gerais also reported the preferential use of Oxic Dystrudepts for subsistence farming, especially banana plantations, due to higher chemical fertility combined with greater soil depth with latosolic morphology and Cambic character. The dystrophic properties of CXbd could be related to the weathering of gneiss-granite rock fragments in the profile. The origin of this soil is probably a mixture of pre-weathered colluvial materials (latosolic oxic), with abundant grains of recently added primary minerals, related to those from upstream rocky outcrops. The natural fertility of the soils in this environment is an important factor that benefits familiar agriculture, reducing
Table 6 - Semi-quantification of minerals in the clay fraction by allocation based on oxide content determined by sulfuric acid attack.

\begin{tabular}{|c|c|c|c|c|c|c|}
\hline \multirow{2}{*}{ Soil } & \multirow{2}{*}{ Horiz. } & \multicolumn{5}{|c|}{ Mineral composition } \\
\hline & & $\mathrm{Kt}$ & $\mathrm{Gb}$ & Gt & $\mathrm{Hm}$ & $\mathrm{Mi}$ \\
\hline & & \multicolumn{5}{|c|}{ dag kg-1 } \\
\hline RLi & 0 & 41.66 & 1.83 & 0.99 & 0.00 & 1.23 \\
\hline PVd & Bt2 & 54.04 & 15.12 & 1.60 & 3.01 & 3.08 \\
\hline LVd & Bw2 & 48.17 & 19.83 & 1.76 & 3.32 & 0.00 \\
\hline CXbd & $\mathrm{Bi}$ & 48.99 & 17.89 & 1.87 & 0.00 & 1.23 \\
\hline RYbd & $\mathrm{C} 1$ & 49.86 & 7.86 & 3.58 & 0.00 & 6.31 \\
\hline GXbd & Cg1 & 29.85 & 14.75 & 3.77 & 0.00 & 22.00 \\
\hline PAd & Bt2 & 50.34 & 17.61 & 7.26 & 0.00 & 0.00 \\
\hline LAd & Bw2 & 34.41 & 17.14 & 5.37 & 0.00 & 0.00 \\
\hline
\end{tabular}

the need for inputs, especially chemical fertilizer, which is rarely used by farmers in the micro-watersheds.

The low levels of exchangeable bases of the Lithic Udifolist (RLi) indicate poor levels of nutrients from the original material. This outcome is explained by both the accentuated slope of the land and the hot/humid climate, which result in intense weathering and accelerated leaching of bases and silica from this profile. In this soil, the presence of a histic horizon is due to the low decomposition of organic matter. It is possibly associated with low availability of nutrients and high levels of exchangeable aluminum, which may contribute to limited microbial activity.

Close to the surfaces of the soils studied, available $P$ levels increase slightly due to the higher levels of organic matter. Similarly, levels of all exchangeable bases 
also increase showing the important influence of the organic matter on the recycling of the nutrients. In general, the A horizons of all soils showed low levels of phosphate adsorption capacity, as inferred by high values of the remaining $\mathrm{P}$ in solution (Prem). These results follow a trend that accompany the increase in organic matter in the surface horizons, evidencing competition of organic anions and phosphates for adsorption sites on the surface of iron and aluminum oxides, in line with results of Oades et al. (1989). On the other hand, B-horizons of all soils showed lower levels of remaining phosphorus (Prem) evidencing a higher power of adsorbing $\mathrm{P}$, influenced by the amounts of clay and oxides in these horizons. There is a direct relationship between higher phosphate adsorption capacity and both clay minerals and $\mathrm{Fe}$ and $\mathrm{Al}$ oxides contents of the soil (Fontes and Weed, 1996).

The higher values of available-P in RYbd are probably associated with the parent material. These soils are derived from sediments originating from areas where steep slopes with younger soils predominate. Therefore, they were formed from less weathered materials and present significant texture variations along the profile. In this way, the availability of $\mathrm{P}$ may be more related to the horizon's texture and to the younger material than to the organic matter content. The release of inorganic $\mathrm{P}$ in more weathered soils depends on fluctuations in the redox conditions (Liptzin and Silver, 2009). On the other hand, labile inorganic $\mathrm{P}$ is higher in younger soils as Neosols mainly due to rapid weathering of parent material in these soils (Yang et al., 2013).

The lower values of exchangeable $\mathrm{Al}^{3+}$ in the surface horizons are due to the known effect of organic matter as an Al complexing agent (Bloom et al., 1979) and to the higher $\mathrm{pH}$ found in these horizons, which precipitates the $\mathrm{Al}$ in solution (Lindsay, 1979).

\section{Sulfuric acid digestion}

The lowest values for the molecular ratio $\mathrm{SiO}_{2} /$ $\mathrm{Al}_{2} \mathrm{O}_{3}(\mathrm{Ki})$, between 1 and 2, were found in the $\mathrm{B}$ horizons of all the profiles, especially the $\mathrm{B}$ horizons of the Oxisols and one Hapludult which has latosolic features (LAd, PAd and LVd). Molecular ratios $>1$ and $<2$ indicate a predominance of kaolinite in the clay fraction mineralogy but the gibbsite is already present in the suite of minerals and as its quantities increase $\mathrm{Ki}$ decreases. However, none of the Ki values reached very low values which would be indicative of gibbsitic mineralogy, according to Embrapa (2013). For younger soils such as RLi, CXbd, RYbd and GXbd, the relatively low Ki values $(<2.00)$ indicate that these soils have had considerable weathering and soil development, probably as a function of the climatic conditions. There is no plausible explanation for the highest Ki values found in the surface horizons. A suggested interpretation is the fact that probably that sulfuric acid digestion would underestimate the concentrations of $\mathrm{Al}_{2} \mathrm{O}_{3}$ from organic matter enriched horizons. If that is the case, care should be taken when using these estimates.
The lower $\mathrm{Fe}_{2} \mathrm{O}_{3}$ and $\mathrm{TiO}_{2}$ values, especially for the Dystrudept (CXbd), suggest that these soils derive from gneiss-granite rock that was poor in ferromagnesian minerals. This indicates corroboration of the occurrence of granitoid gneisses in the region of Guarapari (MME, 1983). In spite of indications that the original material was poorer, the higher levels of exchangeable $\mathrm{Ca}^{2+}, \mathrm{Mg}^{2+}$ and $\mathrm{K}^{+}$in $\mathrm{CXbd}$ seem to support the idea that the proximity of the parent material to the soil surface means that nutrients are constantly being released as time progresses, making these soils less dystrophic compared to the other profiles. Inceptisols present an advanced stage of weathering of feldspars, the most common rock components (Pinto et al., 2015). However, in the CXbd profile a mixture of pre-weathered (latossolic) materials is common, with abundant rock fragments and grains of primary minerals of recent contribution. These coarser fractions of the soil represent the main nutrient reserve, such as the release of $\mathrm{K}$, as in Silva et al. (2008).

In the Endoaquent (GXbd) profile, $\mathrm{Fe}_{2} \mathrm{O}_{3}$ levels were similar to those in the other profiles, suggesting that marked removal of $\mathrm{Fe}$ from the system has not occurred, which would justify the yellowish (matrix = 10YR 3/2) rather than the gray coloring that usually appears in this type of soil, due to the reductive environment. Fontes and Carvalho (2005) emphasize that the Munsell notation for the soil color can give good information about relative amounts of iron oxides in highly weathered and developed soils.

The geology of the area indicates that granadabiotite-gneiss and/or hornblende-gneiss and kinzigites (MME, 1983) are the parent material for some of these soils and suggests that the higher total levels of $\mathrm{K}$ in the CXbd, RYbd and GXbd profiles is probably related to the presence of biotite. The higher levels of exchangeable potassium in CXbd is consistent with the higher levels of total potassium in this soil. However, this is not the case for the RYbd and GXbd profiles, which suggests that the reductive environment slowed or impeded biotite breakdown. Leaching of a part of the total $\mathrm{K}$ from the more weathered soils was evident, but this behavior was not observed for $\mathrm{P}$, as indicated by similar total levels of $\mathrm{P}_{2} \mathrm{O}_{5}$ for all profiles, showing that a large proportion of this compound was retained in the system and, thus, not readily available, i.e., most of it adsorbed onto the $\mathrm{Fe}$ and $\mathrm{Al}$ oxides.

\section{Mineralogical characteristics}

\section{Iron oxides by chemical extractions}

The higher levels of poorly crystalline iron oxides in the clay fraction of the Lithic Udifolist (RLi) (Table 3) seems to be associated with the effect of higher levels of organic $\mathrm{C}$ in the soils inhibiting Fe oxide crystallization (Schwertmann and Taylor, 1989). This also means that higher amounts of amorphous oxides would be expected in these soils, which may be associated with the initial weathering state of this soil, meaning that there was a 
limited time for the minerals formed in the weathering process to reach a higher degree of crystallinity (Cornell and Schwertmann, 2003).

In the Endoaquent (GXbd), the higher levels of Feo are related to the hydromorphic environment, in which reductive conditions cause hematite and goethite to dissolve (Macedo and Bryant, 1989; Fontes and Weed, 1991), followed by the neoformation of poorly crystalline forms. The low values of Feo/Fed obtained for the Paleudult, Hapludult, Oxisols, and Dystrudept (PVd, PAd, LVd, LAd and CXbd) profiles are very close to or slightly higher than those found usually $(<0.04)$ in highly weathered non-hydromorphic soils. Good drainage conditions and time (more developed soil) are factors that contribute to the higher crystallinity of the iron oxides in these profiles.

\section{Clay fraction mineralogy}

The X-ray patterns (Figure 2) of all the profiles studied, especially PVd, LVd, RYbd, PAd, GXbd and PAd, showed peaks of gibbsite of considerable reflection intensity. The gibbsite peaks are indicative of good crystallinity in the Oxisol and Hapludult (LVd, PAd and LAd) profiles and can be associated with environmental conditions favoring their formation and stability, especially when related to the relief. According to Herrmann et al. (2007), the relief through its influences in the rainfall, temperature and drainage is an important factor in gibbsite formation. These authors consider that the relief has a combined effect with climate on the formation of gibbsite in Northern Thailand highland soils, due to the decrease in temperature and increase in rainfall with higher altitudes.

In the Limão stream micro-watershed, located at the highest elevation of the coastal uplands and mountains, the relief is predominantly undulating to mountainous, with variation in the landforms, but tending towards convex-convex land surface forms. In this environment, there are rock outcrops in a number of locations, the erosion is slight and relatively deep, more weathered soils (LAd) are predominant, justifying, in large part, the presence of gibbsite in these soils.

In the higher parts of the Oratorio stream microwatershed the landform is predominantly mountainous to sloping, with intense erosion. In this region, shallow or very shallow soils (CXbd and RLi) are the most common, with few areas of deep soil associated with areas of higher stability.

In the CXbd profile, the porous and friable structure typical of Oxisols is consistent with the presence of gibbsite (Figure 2) and justifies the latosolic morphology. The presence of rock close to the surface, rock fragments in the profile, and consequently the presence of micas, interstratified Mi-HIV and hydroxy-interlayered vermiculite justifies its definition as a Dystrudept. The presence of gibbsite as demonstrated by the X-ray patterns in very young soils, such as the Lhitic Udifolist (RLi) and the saprolite (C-horizon) of a Paleudult (PVd), reveals conditions favorable to the formation of gibbsite.
Rossi and Queiroz Neto (2001) also reported orthents that have a lithic contact within $40 \mathrm{~cm}$ of the mineral soil surface with similar mineralogy, containing kaolinite, mica, interlayer vermiculite and gibbsite in soils of the Serra do Mar complex.

Soils containing gibbsite are common, at least in certain areas of the Serra do Mar and Serra da Mantiqueira regions in southeastern Brazil, as observed by Simas et al. (2005), especially since the average annual rainfall is higher than $1,400 \mathrm{~mm}$. In the Guarapari region, despite lower annual rainfall around 1,300 $\mathrm{mm}$, the formation of gibbsite in the soils seems to follow a pattern consistent with not very high rainfall but spread over the year combined with a sandier texture favoring rapid drainage and high desilication, resulting in the presence of gibbsite. Under these conditions, the genesis of gibbsite can occur as a result of the degradation of intermediate silicate clays (Bates, 1962) or by direct formation from the alteration of primary aluminum silicates such as feldspar and/or plagioclase (Clemente and Azevedo, 2007). The presumption that gibbsite was formed under current conditions of climate, terrain and drainage in the area studied does not seem to exclude the possibility of inherited gibbsite, which could have been formed under past pedogenetic conditions that were hotter and allowed for good drainage. The existence of highly weathered soils side by side by with shallow soils and slopes of bare rock could indicate relics of a more profoundly weathered mantle that covered the area in the past. Occurrence of dissected and ravine slopes, with frequent outcrops of rocks, adjacent to the latosolic mantle, was also reported by Albuquerque Filho et al. (2008) in another Atlantic Forest area.

The X-ray patterns showed similarities between the mineralogy of the Oxyaquic Udifluvent (RYbd) and the Endoaquent (GXbd) profiles and the higher elevation soils (Oxisols and Dystrudepts), evidencing the inheritance of pre-weathered material. The high-intensity peaks of gibbsite in this hydromorphic soil (GXbd), with conditions unfavorable to its genesis, support this hypothesis. On the other hand, the presence of $2: 1$ minerals (illite and vermiculite) in the Oxyaquic Udifluvent (RYbd) and the Endoaquent (GXbd) reflects the possible transport of sediments originating from younger, shallower soils located on steep slopes and even rock outcrops. The presence of gibbsite and minerals such as mica, Mi-HIV and HIV in RYbd and GXbd suggest that the mixture of sediments originated from the different soils upstream, from the most weathered to the youngest, from the pedological point of view. The mixing of different types of parent materials may result in different types of clay minerals in soils (Sulieman et al., 2016).

\section{Conclusions}

The soils of the micro-watersheds studied are generally acid and of low fertility with the Inceptisols exhibiting lower dystrophy, and, in particular higher $\mathrm{K}$ 
availability which, combined with good effective depth conferred by their latosolic character, are two of the reasons that make them the preferred choice for cultivating bananas.

Kaolinite, gibbsite and goethite are, in general, present in the clay fraction of the soils studied and kaolinitic mineralogy is predominant, as indicated by the $\mathrm{Ki}$ indices and the X-ray patterns. Phyllosilicate minerals such as mica, illite, interstratified Mi-HIV and hydroxy-interlayered vermiculite were present only in the less developed soils (RLi, CXbd, RYbd and PVd).

The X-ray patterns revealed the presence of relatively intense gibbsite peaks, even in the very shallow soils in the more rejuvenated landscape, part of the Oratório stream micro-watershed, indicating high rates of both pedogenesis and erosion. It suggests that this area in the past may have had favorable climate, relief and drainage conditions for the formation and stabilization of gibbsite.

\section{Authors' Contributions}

Conceptualization: Cunha, A.M., Fontes, M.P.F., Lani, J.L. Data acquisition: Cunha, A.M. Data analysis: Cunha, A.M. Design of methodology: Cunha, A.M., Fontes, M.P.F., Lani, J.L. Writing and editing: Cunha, A.M., Fontes, M.P.F., Lani, J.L.

\section{References}

Albuquerque Filho, M.R.; Muggler, C.C.; Schaefer, C.E.G.R.; Ker, J.C.; Santos, F.C. 2008. Soils with latosolic morphology and cambic properties in Governador Valadares, middle Rio Doce river, Minas Gerais region, Brazil: genesis and micromorphology. Revista Brasileira de Ciência do Solo 32: 259-270 (in Portuguese, with abstract in English).

Anderson, J.V. 1963. An improved pretreatment for mineralogical analysis of samples containing organic matter. Clays and Clay Minerals 10: 380-388.

Bates, T.F. 1962. Halloysite and gibbsite formation in Hawaii. Clays and Clay Minerals 9: 315: 328.

Bloom, P.R.; McBride, M.B.; Weaver, R.M. 1979. Aluminum organic matter in acid soils: buffering and solution aluminum activity. Soil Science Society of America Journal 43: 488-493.

Chen, P.Y. 1977. Table of key lines in X-Ray powder diffraction patterns of minerals in clays and associated rocks. Geological Survey, Bloomington, IN, USA. Department of Natural Resources Geological Survey Occasional Paper, 21.

Clemente, C.A.; Azevedo, A.C. 2007. Mineral weathering in acid saprolites from subtropical, southern Brazil. Scientia Agricola 64: 601-607.

Coffin, D.E. 1963. A method for the determination of free iron in soils and clays. Canadian Journal of Soil Science 43: 7-17.

Comissão de Fertilidade do Solo do Estado de Minas Gerais [CFSEMG]. 1999. Recommendations for the use of correctives and fertilizers in Minas Gerais = Recomendações para o uso de corretivos e fertilizantes em Minas Gerais. CFSEMG, Lavras, MG, Brazil (in Portuguese).
Cornell, R.M.; Schwertmann, U. 2003. The Iron Oxides: structure, properties, reactions, occurrences and uses, 2ed. Wiley-VCH Verlag GmbH \& Co. Weinheim, Germany.

Empresa Brasileira de Pesquisa Agropecuária [EMBRAPA]. 1978. Reconnaissance Soil Survey of Espírito Santo State = Levantamento de Reconhecimento dos Solos do Estado do Espírito Santo. Embrapa-SNLCS, Rio de Janeiro, RJ, Brazil (in Portuguese).

Empresa Brasileira de Pesquisa Agropecuária [EMBRAPA]. 1997. Manual of Methods of Soil Analysis = Manual de Métodos de Análise do Solo. 2ed. Embrapa Solos, Rio de Janeiro, RJ, Brazil (in Portuguese).

Empresa Brasileira de Pesquisa Agropecuária [EMBRAPA]. 2013. Brazilian System of Soil Classification = Sistema Brasileiro de Classificação de Solos. Embrapa Solos, Rio de Janeiro, RJ, Brazil (in Portuguese).

Fontes, M.P.F.; Carvalho, I.A. 2005. Color attributes and mineralogical characteristics, evaluated by radiometry of highly weathered tropical soils. Soil Science Society of America Journal 69: 1162-1172.

Fontes, M.P.F.; Weed, S.B. 1991. Iron oxides in selected Brazilian Oxisols. I. Mineralogy. Soil Science Society of America Journal 55: 1143-1149.

Fontes, M.P.F.; Weed, S.B. 1996. Phosphate adsorption by clays from Brazilian Oxisols: relationships with specific surface area and mineralogy. Geoderma 72: 37-51.

Herrmann, L.; Anongrak, N.; Zarei, M.; Schuler, U.; Spohrer, K. 2007. Factors and processes of gibbsite formation in northern Thailand. Catena 71: 279-291.

Jackson, M.L. 1979. Soil Chemical Analysis: Advanced Course. 2ed. Published by the author, Madison, WI, USA.

Jenny, H. 1941. Factors of Soil Formation. McGraw-Hill, New York, NY, USA.

Joly C.A.; Metzger, J.P.; Tabarelli M. 2014. Experiences from the Brazilian Atlantic forest: ecological findings and conservation initiatives. New Phytologist 204: 469-473.

Lani, J.L.; Rezende, S.B.; Resende, M. 2001. Environmental stratification in the Itapemirim river basin, state of Espírito Santo. Revista Ceres 48: 239-261 (in Portuguese, with abstract in English).

Lindsay, W.L. 1979. Chemical Equilibria in Soils. WileyInterscience, New York, NY, USA.

Liptzin, D.; Silver, W.L. 2009. Effects of carbon additions on iron reduction and phosphorus availability in a humid tropical forest soil. Soil Biology and Biochemistry 41: 1696-1702.

Macedo, J.; Bryant, R.B. 1989. Preferential microbial reduction of hematite over goethite in a Brazilian Oxisol. Soil Science Society of America Journal 53: 1114-1118.

McKeague, J.A.; Day, J.H. 1966. Dithionite and oxalate extractable $\mathrm{Fe}$ and $\mathrm{Al}$ as aid in differentiating various classes of soils. Canadian Journal of Soil Science 46: 13-22.

Ministério de Minas e Energia [MME]. 1983. Project RADAMBRASIL: maps SF 23/24 Rio de Janeiro/Vitória: geology, geomorphology, pedology, vegetation and land potential use = Projeto RADAMBRASIL: Mapas SF 23/24 Rio de Janeiro/ Vitória: geologia, geomorfologia, pedologia, vegetação e uso do potencial de terra. MME, Rio de Janeiro, RJ, Brazil (in Portuguese). 
Morellato, L.P.C.; Haddad, C.F.B. 2000. Introduction: the Brazilian Atlantic forest. Biotropica 32: 786-792.

Nascimento, P.C.; Lani, J.L.; Zoffoli, H.J.O. 2013. Characterization, classification, and genesis of hydromorphic soils in coastal regions of the state of Espírito Santo, Brazil. Científica 41: 8293 (in Portuguese, with abstract in English).

Nettesheim, C.F.; Menezes L.F.T.; Carvalho, D.C.; Conde, M.M.S.; Araujo, D.S.D. 2010. Influence of environmental variation on Atlantic forest tree-shrub-layer phytogeography in southeast Brazil. Acta Botanica Brasilica 24: 369-377.

Oades, J.M.; Gillman, G.P.; Uehara, G. 1989. Interactions of organic matter and variable-charge clays. p. 69-95. In: Coleman, D.C.; Oades, J.M.; Uehara, G., eds. Dynamics of soil organic matter in tropical ecosystems. NifTAL Project, Honolulu, HI, USA.

Pinto, L.C.; Zinn, Y.L.; Mello, C.R.; Owens, P.R.; Norton L.D.; Curi, N. 2015. Micromorphology and pedogenesis of mountainous Inceptisols in the Mantiqueira range (MG). Ciência e Agrotecnologia. 39: 455-462.

Resende, M.; Bahia Filho, A.F.C.; Braga, J.M. 1987. Clay mineralogy of Latosols estimated by chemical allocation of total oxides content by $\mathrm{H}_{2} \mathrm{SO}_{4}$ digestion. Revista Brasileira de Ciência do Solo 11: 17-23 (in Portuguese, with abstract in English).

Robinson, S.J.B.; van den Berg, E.; Meirelles, G.S.; Ostle N. 2015. Factors influencing early secondary succession and ecosystem carbon stocks in Brazilian Atlantic forest. Biodiversity and Conservation 24: 2273-2291.

Rossi, M.; Queiroz Neto, J.P. 2001. Soils as indicators of the relationships between continental and marine sediments on the coastal plain: Rio Guaratuba (SP). Revista Brasileira de Ciência do Solo 25: 113-120 (in Portuguese, with abstract in English).

Santos, R.D.; Lemos, R.C.; Santos, H.G.; Ker, J.C.; Anjos, L.H.C. 2005. Manual of description and collection of soil in the field = Manual de descrição e coleta de solo no campo. 5ed. Sociedade Brasileira de Ciência do Solo, Viçosa, MG, Brazil (in Portuguese).
Schwertmann, U.; Taylor, R.M. 1989. Iron oxides. p. 380-427. In: Dixon, J.B.; Weed, S.B., eds. Minerals in soil environments. 2ed. Soil Science Society of America, Madison, WI, USA.

Silva, V.A.; Marchi, G.; Guilherme, L.R.G.; Lima, J.M.; Nogueira, F; Guimarães, P.T.G. 2008. Kinetics of K release from soils of Brazilian coffee regions: effect of organic acids. Revista Brasileira de Ciência do Solo. 32: 533-540.

Simas, F.N.B.; Schaefer, C.E.G.R.; Fernandes Filho, E.I.; Chagas, A.C.; Brandão, P.C. 2005. Chemistry, mineralogy and micropedology of highland soils on crystalline rocks of the Serra da Mantiqueira, southeastern Brazil. Geoderma 125: 187-201.

Soil Survey Staff. 2014. Keys to Soil Taxonomy, 12ed. USDANatural Resources Conservation Service, Washington, DC, USA.

Sulieman, M.M.; Ibrahim, I.S.; Elfaki, J.T. 2016. Genesis and classification of some soils of the River Nile terraces: a case study of Khartoum North, Sudan. Journal of Geoscience and Environment Protection 4: 1-16.

Walkley, A.; Black, I.A. 1934. An examination of the Degtjareff method for determining soil organic matter and a proposed modification of the chromic acid titration method. Soil Science 34: 29-38,

Whittig, L.D.; Allardice, W.R. 1986. X-ray diffraction techniques. p. 331-361. In: Klute, A., ed. Methods of soil analysis. 2ed. American Society of America, Madison, WI, USA.

Yang, X.; Post, W.M.; Thornton, P.E.; Jain, A. 2013. The distribution of soil phosphorus for global biogeochemical modeling. Biogeosciences 10: 2525-2537.

Zangrande, M.B.; Rezende, S.B. 1989. Characteristics of a Red Yellow Podzolic of the coastal plateaus of the north of the state of Espírito Santo. Revista Ceres 36: 147-172 (in Portuguese, with abstract in English). 